\title{
TITLE
}

\section{Regulatory identification of BPA as an endocrine disruptor: context and methodology}

\section{AUTHORS}

Beausoleil $\mathrm{C}^{1}$, Emond $\mathrm{C}^{2 *}$, Cravedi $\mathrm{JP}^{3 *}$, Antignac $\mathrm{JP}^{4 *}$, Applanat $\mathrm{M}^{5 *}$, Appenzeller $\mathrm{BMR}^{6 *}$ Beaudouin $\mathrm{R}^{7 *}$, Belzunces $L P^{8^{*}}$, Canivenc-Lavier $M C^{9 *}$, Chevalier $\mathrm{N}^{10^{*}}$, Chevrier $\mathrm{C}^{11^{*}}$, Elefant $\mathrm{E}^{12^{*}}$, Eustache $\mathrm{F}^{13^{*}}$, Habert $\mathrm{R}^{14^{*}}$, Kolf-Clauw $\mathrm{M}^{15^{*}}$, Le Magueresse-Battistoni $\mathrm{B}^{16^{\prime}}$, Mhaouty-Kodja $\mathrm{S}^{17^{*}}$, Minier $\mathrm{C}^{18^{*}}$, Multigner $\mathrm{L}^{11^{*}}$, Schroeder $\mathrm{H}^{19 *}$, Thonneau $\mathrm{P}^{20 *}$, Viguié $\mathrm{C}^{21^{*}}$, Pouzaud $\mathrm{F}^{1}$, Ormsby $\mathrm{JN}^{1}$, Rousselle $\mathrm{C}^{1}$, Verines-Jouin $\mathrm{L}^{1}$, Pasquier $\mathrm{E}^{1}$, Michel $C^{1}$

*experts member of ANSES's Thematic Working Group on Endocrine disruptors

${ }^{1}$ ANSES, Risk Assessment Department, Maisons-Alfort, France

${ }^{2}$ DSEST, School of public health, University of Montreal, Quebec, Canada

${ }^{3}$ INRA, Toxalim, UMR1331, Toulouse, France France

${ }^{4}$ LABERCA, UMR 1329, INRA-Oniris Nantes, France

${ }^{5}$ CNRS, France

${ }^{6}$ Human Biomonitoring research Unit, Luxembourg Institute of Health, Luxembourg

${ }^{7}$ INERIS, Unité Modèles pour I'Ecotoxicologie et la Toxicologie (METO), Parc ALATA BP2, 60550 Verneuil en Halatte, France

${ }^{8}$ INRA, Laboratoire de Toxicologie Environnementale, UR 406 A\&E, CS 40509, 84914 Avignon Cedex 9, France

${ }^{9}$ Centre des Sciences du Goût et de I'Alimentation, INRA, CNRS, agrosup, Université de Bourgogne - Franche-Comté, Dijon, 21000, France.

10 University Hospital of Nice, France \& INSERM UMR U1065 - Centre Méditerranéen de Médecine Moléculaire, Nice, France

${ }^{11}$ Univ Rennes, Inserm, EHESP, Irset (Institut de recherche en santé, environnement et travail) - UMR_S 1085, F-35000 Rennes, France

${ }^{12}$ Centre de Référence sur les Agents Tératogènes (CRAT), AP-HP Armand Trousseau Hospital, Paris, France

13 CECOS, AP-HP, Paris Seine-Saint-Denis University Hospitals, Jean Verdier Hospital, Paris

${ }^{14}$ Genetic stability, stem cells and radiations, CEA, INSERM U 967, University ParisDiderot, CEA research center, 92265 Fontenay aux Roses, France

15 CREFRE, Toulouse University, INSERM, Toulouse Veterinary School, 23 chemin des Capelles, BP 87614, F310176 Toulouse Cedex3., France

16 Univ-Lyon, CarMeN laboratory, INSERM U1060, INRA U1397, Université Claude Bernard Lyon1, INSA Lyon, Charles Mérieux Medical School, F-69600 Oullins, France

17 Sorbonne Universités, UPMC Univ Paris 06, INSERM, CNRS, Neuroscience Paris Seine Institut de Biologie Paris Seine, 75005 Paris, France

\footnotetext{
1 Abbreviations: ANSES: Agence Nationale de Sécurité Sanitaire de l'alimentation, de l'environnement et du travail (French Agency for Food, Environmental and Occupational Health \& Safety) ; AOP: adverse outcome pathway; BPA: bisphenol A; CD: compact disc; CLP: Classification, Labelling and Packaging; CMR: Carcinogen, Mutagen, Reprotoxic; DES: diethylstilbestrol; $\mathrm{E}_{2}: 17-\beta$ estradiol; EC: European Commission; ECHA: European Chemical Agency; ED: endocrine disruptor; EDC: endocrine disrupting chemical; EZH2: Enhancer of Zeste Homolog 2; HOTAIR: HOX transcript antisense RNA; JRC: Joint Research Center, the European Commission's in-house science service; MOA: mode of action; OECD: Organisation for Economic Co-operation and Development; REACH: Registration, Evaluation, Authorisation and Restriction of Chemicals; SVHC: substance of very high concern; WHO: World Health Organization; WoE: weight of evidence
} 
${ }^{18}$ Laboratory of Ecotoxicology, UMR INERIS SEBio, Normandie Université, BP 540, 76058 Le Havre, France

19 Calbinotox, Faculté des Sciences et Technologies, Université de Lorraine, 54500, Vandoeuvre les Nancy, France

${ }^{20}$ INSERM, France

21 Toxalim (Research Centre in Food Toxicology), INRA, ENVT, INP-Purpan, UPS, Toulouse, France

Corresponding author: Elodie Pasquier, ANSES, 14 rue Pierre et Marie Curie, F-94701 Maisons-Alfort Cedex, France, elodie.pasquier@anses.fr

\section{KEYWORDS}

Bisphenol A, endocrine disruption, ED, SVHC, Substance of Very High Concern, REACh

\section{FUNDING/Declaration of interest}

This review was carried out in the framework of assessments performed by the French Agency for Food, Environmental and Occupational Health and Safety (ANSES). The authors declare no conflict of interest.

\section{HIGHLIGHT}

- $\quad$ BPA is an ubiquitous substance due to its multiple and large uses

- BPA is closely associated with ongoing controversies related to ED

- Regulation of ED is in its early stages and requires a high level of evidence

- Several ED-mediated effects of BPA achieve these requirements

\section{ABSTRACT (150 words)}

BPA is one of the most investigated substances for its endocrine disruptor (ED) properties and it is at the same time in the center of many ED-related controversies, the analysis on how BPA fits to the regulatory identification as an ED is a challenge in terms of methodology. It is also a great opportunity to test the regulatory framework with a uniquely data-rich substance and learn valuable lessons for future cases. From this extensive database, it was considered important to engage in a detailed analysis so as to provide specific and strong evidences of ED while reflecting accurately the complexity of the response as well the multiplicity of adverse effects. An appropriate delineation of the scope of the analysis was therefore critical. Four effects namely, alterations of estrous cyclicity, mammary gland development, brain development and memory function, and metabolism, were considered to provide solid evidence of ED-mediated effects of BPA.

This introductory article of the Special issue: Is BPA an ED? aims to present the regulatory identification of BPA as an endocrine disruptor, the framework of this analysis and how relevant adverse effects were selected. The database analysis was performed in close collaboration with the ANSES' Thematic Working group on Endocrine Disruptors with an analysis focused on effects relevant for human health

Since specific definition of what an endocrine disruptor is was still under debate at the EU level, the WHO/IPCS (2002) definition of an endocrine disruptor (ED), widely accepted and associating several mandatory components, were considered in this review: "an endocrine disruptor is an exogenous substance or mixture that alters 
function(s) of the endocrine system and consequently causes adverse health effects in an intact organism, or its progeny, or (sub)populations." Lastly, the adverse effects of BPA to be further analysed were selected when relevant evidence of an ED MoA and an adverse effect were identified with a sufficient level of evidence. In addition, the plausible link between the adverse effects and the endocrine MoA were thoroughly considered as well as the human relevance.

\section{A widely use chemical despite early signals}

Bisphenol A (BPA, see Table 1 for its main identifiers) was first synthetized in 1891 by the Russian chemist Alexander Dianin (Dianin, 1981). The estrogenic properties of Bisphenol A (BPA) have been discovered in the 1930's (Dodds and Lawson, 1936) when scientists were searching for synthetic chemicals that could replace the expensive natural estrogen in pharmacological applications. They identified BPA as a weak functional estrogen compared to other compounds such as diethylstilbestrol (DES): BPA induced persistent vaginal cornification in ovariectomized rats exposed to daily injections of $188 \mathrm{mg}$ of BPA $/ \mathrm{kg} / \mathrm{d}$ diluted in sesame oil for 3 days while a similar effect was obtained from $0.76 \mu \mathrm{g} / \mathrm{kg} / \mathrm{d}$ of DES under similar experimental conditions (Dodds and Lawson, 1938). Consequently the use of BPA as a drug has not been further developed. However, BPA has found applications in several important other domains.

Under the process of the REACh regulation, the total tonnage registered for BPA is comprised between 1000000 and 10000000 metric tonnes per year, as declared by registrants in the registration dossiers available on the free website of the European Chemicals Agency (ECHA).

BPA has been used for more than 50 years mainly as a monomer in the production of polycarbonate plastics and as an intermediate in the synthesis of epoxy resins. It is also used as a component in polyester resins, polysulfone resins and polyacrylate resins. Polycarbonates are used in the composition of different objects sucha as CDs, glasses, plastic bottles, baby bottles, etc., while epoxy resins are principally used to coat the inside of food tins and in dental fillings. BPA is also used as a developer in thermal papers (ANSES, 2013), in the synthesis of others polymers such as polyester carbonate (automotive and transport, consumers products, etc), polyarylates (medical equipment, automotive, aeronautics, etc), polysulfones (medical equipment, domestic appliances, etc), polyetherimides (electric and electrical equipment, aeronautical, etc) polyols (used in the production of polyurethane), etc. BPA can also be employed to produce other resins such as vinyl ester resins (automotive and marine construction, etc), phenolic plastic resins (abrasives, insulation, etc), unsaturared resins (bisphenol fumarates, BPA epoxy dimethacrylates). BPA can also be used to synthesize flame retardants found for example in consumer products (computers, television, dishwater, etc) (ANSES, 2011). 
Table 1: Substance identity

\begin{tabular}{|l|l|}
\hline Systematic chemical name: & $4,4^{\prime}$-isopropylidenediphenol \\
\hline Synonyms: & $\begin{array}{l}\text { Bisphenol } \mathrm{A} \\
\text { BPA } \\
\text { phenol, 4,4'-(1-methylethylidene)bis- } \\
4,4^{\prime} \text {-propane-2,2-diyldiphenol } \\
2,2 \text {-bis(4-hydroxyphenyl)propane }\end{array}$ \\
\hline CAS number: & $80-05-7$ \\
\hline EC number: & $201-245-8$ \\
\hline Molecular formula: & $\mathrm{C}_{15} \mathrm{H}_{16} \mathrm{O}_{2}$ \\
\hline Structural formula &
\end{tabular}

In relation to its wide use in many products, the presence of BPA in environment is ubiquitous and contributes to a broad and nearly continuous human exposure (Chevalier et al., 2015; Legeay et al., 2017).

Contamination of food by food containers is considered one of the most important source of exposure to BPA by the oral route (ANSES, 2013 and 2014; EFSA, 2015). Drinking water may also be contaminated by BPA, at concentrations ranging from 0.014 to $0.317 \mu \mathrm{g} / \mathrm{L}$, by its release from epoxy resin lining of pipes Arnold et al., 2013). Dust inhalation and dermal contact with thermal papers are also a significant source of human exposure (Michalowicz, 2014). In addition, BPA may also be present in cosmetics, toys, medical equipments and more.

The consequence of its ubiquitous presence result in a universal human exposure with more than $95 \%$ of all people that having BPA in their bodies (Calafat, 2005). BPA is found in human serum and urine. In addition, its presence in amniotic fuid and umbilical cord blood reflects maternal as well as fetal exposure (Vandenberg et al., 2007).

\section{BPA is associated with the emergence of controversies in the field of endocrine disruption}

The concern that BPA may induce adverse effects through endocrine disruption (ED) has been raised for decades. In parallel, intense debates have emerged questioning whether toxicity of EDs is only influenced by the level of the dose administered, as traditionally characterized in particular in the regulatory context (Vandenberg et al., 2009). The importance of critical periods of vulnerability to xenobiotics during development has been emphasized. The proximity and interactions between various endocrine systems and negative feedback loop, depending on dose levels may have large implications in the complexity of the toxic response. The controversy around these concepts has been notably exemplified in the abundant literature addressing BPA's pattern of toxicity. The complexity of BPA action raises difficulties in the characterization of its toxic response, as well as regulatory consideration and acceptance, and it may be interpreted as 
inconsistencies that challenge the acknowledgement of the effect itself. In particular, different toxic responses are reported across various animal models oftenly exhibiting sex-dependencyFinally regulatory standardized studies may not address appropriately all relevant endpoints and effects in relation to ED properties (Myers et al., 2009; Schug et al., 2015).

An endocrine disruptive contribution has been suggested for several public health issues that are of major attention in western countries due to their prevalence and claimed increasing trends, i.e. reproductive disorders, hormonal-dependent cancers, metabolic diseases, neurobehavioural disorders. Together with concerns raised by possible limitations of existing framework for hazard characterization and risk management, it has contributed to identify the need of a specific definition of an endocrine disruptor as a first step to target specific regulatory measures.

\section{Regulatory consideration of ED}

Several European regulations have been among the first to address ED. In particular, according to the Regulations of the Biocidal Products and Plant Protection Products, active substances which are identified as ED are not allowed on the EU market unless specific conditions are met. Scientific criteria to identify substances which have endocrine disrupting properties in this regulatory framework need however to be developed to allow an appropriate application of these regulations. Finalisation of the criteria has however not been successful until now despite the legal of obligation for the European Commission to adopt criteria by December 2013 (EC, 2016) and scientific and political debates are still ongoing.

Such criteria are not mandatory to address ED substances in the REACH regulation. Having endocrine disrupting properties is one of the possible basis to identify a substance as a Substance of Very High Concern (SVHC), with the condition that its ED properties are demonstrated to give rise to a level of concern equivalent to a carcinogen, mutagen or reproductive toxicant (CMR). Identifying a substance as an SVHC opens the possibility for a stringent risk management measure that conditions the obtention of its temporary authorisations and strongly encourages its substitution.

BPA is in the scope of the REACH Regulation and its ED properties have been extensively investigated. A detailed analysis of the BPA database has been performed to define which effects of BPA indeed fulfil the requirements for its regulatory identification as an SVHC due to its ED properties. This analysis was performed by ANSES's Thematic Working Group on Endocrine disruptors (ANSES, 2017) and is depicted in this special issue. How each selected effect is considered to be mediated by an ED MoA is detailed in the succession of articles in this special issue.

\section{Application to the singular case of BPA}

Because BPA is a very documented and debated endocrine disrupting chemical (EDC), the analysis on how BPA fits to the regulatory identification as an ED is a challenge in terms of methodology. It is a great opportunity to test the regulatory framework with a unique data-rich substance and learn valuable lessons for future cases. From the extensive BPA database, it was considered important to engage in a detailed analysis so as to provide specific and strong evidences and build a solid case for ED identification that reflects accurately the complexity of the response as well as the multiplicity of adverse effects that are at stake. An appropriate delineation of the scope of the analysis was therefore critical. In particular, it was decided to focus the analysis on effects relevant for human health and not to address effects on species from the environment. Many basic endocrine regulations are however widely conserved across species, although with great variations in the degree of complexity and as a general rule, a comprehensive 
analysis of all potential human and non-human targets is considered beneficial to provide consistency in the assessment. However, in the case of BPA, the environmental exposure and related target effects were excluded from the scope of the analysis in the sake of an efficient use of ressources.

\subsection{Framework of the analysis and selection of relevant adverse effects}

The WHO/IPCS (2002) definition of an ED is widely accepted and associates several mandatory components: "an endocrine disruptor is an exogenous substance or mixture that alters function(s) of the endocrine system and consequently causes adverse health effects in an intact organism, or its progeny, or (sub)populations."

In line with this definition, the European Commission's Endocrine Disrupters Expert Advisory group agreed in 2013 "that the elements for identification of an endocrine disrupter were demonstration of an adverse effect for which there was convincing evidence of a biologically plausible causal link to an endocrine disrupting mode of action and for which disruption of the endocrine system was not a secondary consequence of other non-endocrine-mediated systemic toxicity. Relevance of the data to humans should be assumed in the absence of appropriate data demonstrating non-relevance." (JRC 2013)

Based on the definition given by WHO of an adverse effect a change in the morphology, physiology, growth, development, reproduction, or life span of an organism, system, or (sub)population that results in an impairment of functional capacity, an impairment of the capacity to compensate for additional stress, or an increase in susceptibility to other influences (WHO/IPCS 2009) it has been decided to built our proposal on adverse effects previously recognized through existing regulatory acceptance. Indeed, it was decided to further consider relevant (i) adverse effects acknowledged by a harmonised classification according to the CLP Regulation, i.e. BPA's effects on fertility (ECHA, 2014), or (ii) adverse effects considered for risk assessment in a proposal to restrict the use of BPA in thermal paper i.e., in addition to general toxicity on liver and kidney, effects on brain and behavior, female reproductive system, metabolism and obesity, immunotoxicity and mammary gland were considered in the hazard, risk and health impact assessments. Derivation of the toxicological reference dose was based on kidney effects. For the other effects, it was concluded that although they do not allow a quantification of the doseresponse relationships, these effects shall be accounted for through the setting of Assessment Factors (ECHA, 2015).

It is assumed in this analysis that a substance should fulfill the recommendations from the European Commission's Endocrine Disrupters Expert Advisory group outlined above in order to be identified as an endocrine disruptor under REACH. BPA's database has accordingly been assessed based on identification of (i) adverse health effects, (ii) endocrine mode of action (MoA), (iii) plausible link between adverse effects and endocrine MoA, and (iv) human relevance.

It is important to emphasize that, as discussed in Kortenkamp et al. (2012), there is a clear distinction between the terms MoA and mechanism of action. The mechanism of action is typically defined as the totality of mechanistic steps, whereas the MoA refers to a less detailed sequence of key events. MoA is not intended to build a comprehensive model of a chemical mechanism of action. This is also in line with definitions proposed in the OECD guidance document on adverse outcome pathways (AOP) (OECD, 2013): "MoA differs from mechanism, in that the MoA requires a less detailed understanding of the molecular basis of the toxic effect".

It is therefore considered that for the purpose of identifying an ED, the whole mechanistic steps do not need to be understood. This is particularly critical for the identification of ED's as due to the complexity of mechanisms at stake, ED identification 
would be nearly impossible.

BPA has been shown to trigger various adverse effects on health as reviewed in previous work (ANSES, 2013). An ED MoA is considered to play a substantial role for several of these effects and it is important to consider and analyse in this specific review the scope of effects that are at stake when considering the ED properties and potential health impacts of BPA.

The adverse effects of BPA to be further analysed have been selected considering that there is relevant evidence of an ED MoA and that the adverse effect is identified with a sufficient level of evidence. Adverse effects of BPA were previously discussed in different European regulatory settings further to different ANSES's initiatives.

\subsection{Considerations related to the relevance of data}

The analysis of each adverse effect selected and of its ED MoA was based on literature searches up to May 2016.

Many studies have investigated the effects of BPA by oral route as well as by subcutaneous route of administration. When BPA is administered by subcutaneous route, the daily dose can be controlled with greater accuracy, making it possible to reproduce a regular exposure pattern (see previous ANSES report, 2013). Subcutaneous administration bypasses the digestive barrier, intestinal and/or skin metabolism and the enterohepatic first-pass effect and is generally considered as an artificial route of exposure compared to oral route of exposure with questionable relevance in risk assessment. Indeed, it is generally well accepted that unconjugated BPA is the biologically active form and the data collected in rodents show significantly higher proportions of unconjugated BPA after subcutaneous and intraperitoneal administrations, than in the case of an oral administration (Doerge et al., 2010; Collet, 2012). Although the human experimental database is rather limited (Volkel et al., 2002 and 2005), there is indication that BPA undergoes a high hepatic first-pass effect in humans after oral exposure, resulting in a short half-life ( $<6$ hours) and a low systemic availability of unconjugated BPA. Consideration of subcutaneous studies for human risk assessment would therefore require dose correction.

It needs to be highlighted that other routes of exposure relevant for humanssuch as the dermal route or inhalation also bypass hepatic first-pass. In addition, in rodents and to a lesser extent in humans, the hepatic metabolism capacity in newborns is limited, resulting in a reduced hepatic first-pass effect. Therefore, when developmental or perinatal exposure is considered, the effects observed after subcutaneous exposure are considered fully relevant for the purpose of the present analysis, that is to say hazard identification and MoA considerations.

Studies performed by alternative routes of administration (intracerebral, intraperitoneal) are anecdotal and they were considered only in relation to the analysis of the MoA.

The selection of the studies have not been restricted to specific levels of doses and both "low doses" and "standard doses" for regulatory testing have been considered relevant for the identification of adverse effects and the understanding of the MoA. It is, however, recognised that the MoA may have different patterns and modulations across the whole range of doses.

Finally, although not considered as relevant for the identification of an adverse effect, studies performed in non-intact animals (i.e. ovariectomised animals) were included for the understanding of the MoA. 
Systematic rating of studies using Klimisch scores (Klimisch et al., 1997), as generally recommended in regulatory setting such as $\mathrm{REACH}$, was not considered relevant for the present analysis. Klimisch scores are intended to provide a score in comparison to standard regulatory guidelines. However, several of the specific adverse effects examined in the present analysis (e.g. alteration of the mammary gland development, alteration of neural structures and alteration of insulin production and insulin sensitivity) are not addressed in any specific guideline protocol. In addition, the analysis was focused on studies that preferentially investigate mechanistic aspects and not the adverse effect only. Standard regulatory guideline studies are for this purpose neither required nor generally conducted for practical reason. Very complex protocols would indeed be needed to additionally dig into specific parameters. Finally, for most of the adverse effects, a very large database of studies is available and not all studies provide similar results. Therefore it was considered most relevant to follow a Weight of Evidence (WoE) approach for the present analysis. As defined in ECHA's Practical Guide: How to use alternatives to animal testing (ECHA 2016) "The weight of evidence approach commonly refers to combining evidence from multiple sources to assess a property under consideration". As discussed in the guide, the WoE approach is beneficial when the information from each source individually may be regarded as not sufficient and when several available studies give conflicting results. It also emphasizes that "Expert judgement is vital in the construction and appraisal of the WoE package, namely when considering the reliability, relevance and adequacy, integrating and comparing different pieces of information and assigning a weight to each piece of data."

The present analysis was performed by ANSES in close collaboration with its Thematic Working group on Endocrine Disruptors (ANSES, 2017). Scientific studies considered irrelevant due to major deficiencies in their design and/or reporting were not included in the analysis and are not presented in this review. The studies were considered on the basis of their relevance, reliability and adequacy for the analysis and were qualitatively weighted on the basis of collective expert judgement to produce a conclusion on the selected adverse effects and their ED MoA. Data on Humanwere analysed together. Experimental data were compared to each other with specific consideration of the periods of exposure in particular. The conclusion of the WoE for each effect was based on the combination of human and experimental in vivo and in vitro data.

The ED criteria that have been adopted in EU for PPP and BPD mention that in applying the WoE determination, the assessment of the scientific evidence shall, in particular, consider all of the following criteria: (i) both positive and negative results; (ii) the relevance of the study designs, for the assessment of adverse effects and of the endocrine mode of action; (iii) the quality and consistency of the data, considering the pattern and coherence of the results within and between studies of a similar design and across different species; (iv) the route of exposure, toxicokinetic and metabolism studies; ( $v$ ) the concept of the limit dose, and international guidelines on maximum recommended doses and for assessing confounding effects of excessive toxicity. "Although the WoE approach applied during the present analysis was not formalized in a standardized manner as recently proposed by EFSA, 2017. The WoE approach applied was performed in agreement with the SCENIHR report, 2012 and ECHA guidance, 2010) and all the cited above criteria have been considered for weighing and integrating the piece of evidence for each adverse effect presented.

\subsection{Adverse effects selected to demonstrate ED properties of BPA}

The adverse effects of BPA were fully evaluated under the REACH regulation in a previous ANSES report (ANSES, 2014). The following adverse effects were considered to be established with a sufficient level of evidence: 
(i) effect on the reproductive function:

The effects of BPA on reproduction are multiple and involve alterations of both male and female functions (ANSES, 2014). For the sake of clarity considering the extent of the database, it has been decided to focus the investigation on a more specific effect for which the ED MOA is the most obvious. BPA has been shown in particular to alter estrous cycles. These latter are, by nature, driven through timed hormonal sequences whose alterations most likely involve the disruption of their sequence. The ability of BPA to affect cyclicity of the female reproductive system and how this alteration is exerted through hormonal changes was therefore selected for the purpose of demonstrating that BPA exerts its effects on reproduction through endocrine disruption [see Viguié et al., 2018; This Issue].

(ii) alteration of mammary gland development

Development and function of the mammary gland are endocrine-dependent processes, involving a complex hormonal scheme depending on its developmental stage. Fetal and/or postnatal exposure to BPA disrupts epithelium-stroma interactions in female mammary gland mammals, increases mainly mammary gland's terminal end buds number, lateral branching, ductal and intraductal hyperplasia, , ultimately increasing the susceptibility of the mammary gland to chemical carcinogens (ANSES, 2014). Some of the BPA-effects are reversed by estrogen and/or G-protein-coupled estrogen receptor inhibitors. Lastly, emerging epigenetic studies showed that BPA alters the expression of $\mathrm{E}_{2}$-dependent genes involved in cell proliferation (HOTAIR, EZH2) by modulating their methylation, providing an interpretation of the delayed effect of fetal exposure to BPA [see Applanat et al., 2018; This issue].

\section{(iii) alteration of brain development and memory function}

Many recent studies have confirmed previous results that showan alteration of learning and memory [see Sakina Mhaouty-Kodja et al., 2018; This Special Issue]. Observation of functional impairments of behaviour is strongly supported by the concomitant molecular and cellular changes in brain regions underlying these cognitive processes (ANSES, 2014). In addition, solid evidence of the role of disruption of the estrogenic pathway in these alterations has been produced through the reversal of BPA-induced effects with estrogenic receptor antagonist or by interference of BPA with administered estradiol in ovariectomised animals. The analysis of ED-related neurobehavioural effects of BPA has therefore focused on alteration of memory and learning [see Sakina Mhaouty-Kodja et al., 2018; This Special Issue].

\section{(iv) metabolic alterations}

Metabolic disturbances elicited by BPA include diabetogenic as well as obesogenic effects (ANSES, 2014). More specifically, BPA has been shown to alter insulin synthesis and/or release by $\beta$-pancreatic cells, and insulin signalisation within insulin-sensitive organs (i.e., liver, muscle, adipose tissues). This resulted in variations in the expression of specific hepatic or adipose tissue markers, which are indicative of a state of insulin resistance and considered to be hallmarks of hormonal adverse effects. The analysis of ED-mediated effect of BPA on metabolism has therefore focused on alteration of insulin production and action [see LeMagueresse-Batistoni et al., 2018; This Special Issue].

Overall, these four effects are considered a relevant basis to further investigate how BPA can fulfill the requirements for a regulatory identification as an ED substance.

Separated articles in this issue are dedicated to each of these effects and to the investigation of ED involvement. 
This selection of effects is however not intended to be an exhaustive list of ED-mediated effects of BPA. It is rather the level of evidence of an ED-mediated MoA of BPA for other effects is considered lower. In particular, immunotoxic effects of BPA have also been described (Ménard et al., 2014a and 2014b) and recognized in previous regulatory assessment (ECHA, 2015). The role of estrogens has been often reported in immunocompetence and in the development of innate and adaptive immune response (Fish et al., 2008) and they may also be mediated through an ED MoA. However, the variability of the immunotoxic effects observed makes the interpretation and the transposition of these effects to humans uncertain. The adversity of these effects and their relation to an ED-mediated MoA is not yet clearly demonstrated. A recent review also reports evidences suggesting an effect of BPA on the cardiovascular system that may involve estrogen receptor (Gao et al., 2014). These effects were however not further investigated because the level of evidence was considered insufficient at this point but it is likely that the range of effects related to the ED-properties of BPA may be wider than those selected above. 


\section{References}

ANSES (2011). Health effects of bisphenol A - Knowledge about the uses of bisphenol A.

ANSES (2013). Risk assessment of Bisphenol A (BPA) on human health. March 2013.

ANSES (2014). Annex XV Restriction Report - Proposal for a restriction - 4.4'isopropylidenediphenol (bisphenol A; BPA). May 2014.ANSES (2017). Annex XV report. Proposal for identification of a substance of very high concern on the basis of the criteria set out in REACh Article 57. March, 2017. Available on : https://echa.europa.eu/documents/10162/93bf4be3-9af6-d7ca-8b074e8fb42bad11

Arnold SM, Clark KE, Staples CA, Klecka GM, Dimond SS, Caspers N, et al. (2013) Relevance of drinking water as a source of human exposure to bisphenol A. J Expo Sci Environ Epidemiol. 23(2):137-44

Calafat AM, Kuklenyik Z, Reidy JA, Caudill SP, Ekong J, Needham LL. (2005) Urinary concentrations of bisphenol $A$ and 4-nonylphenol in a human reference population. Environ Health Perspect. 113:391-395

Chevalier N, Fénichel P. (2015) Bisphenol A: Targeting metabolic tissues. Rev Endocr Metab Disord.16(4):299-309. doi: 10.1007/s11154-016-9333-8. Review. PubMed PMID: 26820262.

Collet S. (2012). Développement d'une approche toxicocinétique/toxicodynamique basée sur des mécanismes physiologiques pour évaluer les effets oestrogéniques du Bisphénol A, Thèse, Institut National Polytechnique de Toulouse (INP Toulouse)

Dianin A. (1891) In Russian:"On condensation products of ketones with phenols". Journal of the Russian Physical Chemistry Society, 23 : 488-517, 523-546, 601611 (see especially pages 491-493)

Dodds EC, Lawson W. (1936) Synthetic oestrogenic agents without the Phenanthrene agents. Nature 137, 996

Dodds EC et al. (1938) Oestrogenic Activity of Certain Synthetic Compounds. Nature, $141,247-248$

Doerge DR, Twaddle NC, Vanlandingham M, and Fisher JW. (2010). Pharmacokinetics of bisphenol $A$ in neonatal and adult Sprague-Dawley rats. Toxicology and Applied Pharmacology 247 (2): 158-165

EC (2016). Commission staff working document. Impact assessment. Defining criteria for identifying endocrine disruptors in the context of the implementation of the plant protection products regulation and biocidal products Regulation. Main report. COM (2016) 350 final / SWD (2016) 212 final. http://ec.europa.eu/health//sites/health/files/endocrine disruptors/docs/2016 im pact assessment en.pdf (accessed on 20 July 2017).

ECHA (2010). Practical guide 2: How to report weight of evidence. ECHA-10-B-05-EN. $24 / 03 / 2010$

ECHA (2014). Committee for Risk Assessment (RAC). Opinion proposing harmonised classification and labelling at EU level of Bisphenol A; 4,4'-isopropylidenediphenol. Adopted 14 march 2014. ECHA. Available at http://echa.europa.eu/documents/10162/777918ff-33b5-46ff-be892bdc406d34fa (accessed on 20 July 2017). 
ECHA (2015). Opinion on an Annex XV dossier proposing restrictions on Bisphenol A. Compiled version prepared by the ECHA Secretariat of RAC's opinion (adopted 5 June 2015) and SEAC's opinion (adopted 4 December 2015). https://echa.europa.eu/documents/10162/9ce0977b-3540-4de0-af6d16ad6e78ff20 (accessed on 20 July 2017).

ECHA (2016). Practical Guide: How to use alternatives to animal testing to fulfil your information requirements for REACH registration. Version 2.0 July 2016 https://echa.europa.eu/documents/10162/13655/practical guide how to use al ternatives en.pdf/148b30c7-c186-463c-a898-522a888a4404 (accessed on 20 July 2017).

EFSA (2015). Scientific Opinion on the risks to public health related to the presence of bisphenol A (BPA) in foodstuffs, EFSA journal 2015;13(1):3978.

EFSA (2017). Guidance on the use of the weight of evidence approach in scientific assessments.

Fish EN. (2008) The X-files in immunity: sex-based differences predispose immune responses. Nat. Rev. Immunol, 8:737-744

Gao X, Wang HS. (2014) Impact of bisphenol a on the cardiovascular system epidemiological and experimental evidence and molecular mechanisms. Int J Environ Res Public Health, 11(8):8399-413

JRC (2013). Key Scientific issues relevant to the identification and characterisation of endocrine disrupting substances - Report of the Endocrine Disruptors Expert Advisory Group (ED EAG). Eds. Munn S. and Gourmenou M. Pp 32. Available at: https://ec.europa.eu/jrc/en/publication/eur-scientific-and-technical-researchreports/key-scientific-issues-relevant-identification-and-characterisationendocrine-disrupting (accessed on 20 July 2017).

Klimisch HJ, Andreae M, Tillmann U. (1997) A systematic approach for evaluating the quality of experimental toxicological and ecotoxicological data. Regul Toxicol Pharmacol. 25(1):1-5.

Kortenkamp A. et al. (2012) State of the art assessment of endocrine disrupters. Final report. Available

at http://ec,europa.eu/environment/chemicals/endocrine/pdf/sota edc final report. pdf (accessed on 20 July 2017).

Legeay S, Faure S. (2017) Is Bisphenol A an environmental obesogen? Fundam Clin Pharmacol. doi: $10.1111 /$ fcp.12300. [Epub ahead of print] Review. PubMed PMID: 28622415.

Menard S et al. (2014a) Food intolerance at adulthood after perinatal exposure to the endocrine disruptor bisphenol A. FASEB J, 28(11):4893-900.

Menard S et al. (2014b). Perinatal exposure to a low dose of bisphenol A impaired systemic cellular immune response and predisposes young rats to intestinal parasitic infection. PLoS One, 9(11):e112752.

Michałowicz J. (2014) Bisphenol A--sources, toxicity and biotransformation. Environ Toxicol Pharmacol. 37(2):738-58. doi: 10.1016/j.etap.2014.02.003. Epub 2014 Feb 8. Review. PubMed PMID: 24632011.

Myers JP, vom Saal FS, Akingbemi BT, Arizono K, Belcher S, Colborn T, Chahoud I, Crain DA, Farabollini F, Guillette LJ Jr, Hassold T, Ho SM, Hunt PA, Iguchi T, Jobling S, Kanno J, Laufer $H$, Marcus M, McLachlan JA, Nadal A, Oehlmann J, Olea N, Palanza P, Parmigiani S, Rubin BS, Schoenfelder G, Sonnenschein C, Soto AM, 
Talsness CE, Taylor JA, Vandenberg LN, Vandenbergh JG, Vogel S, Watson CS, Welshons WV, Zoeller RT. (2009) Why public health agencies cannot depend on good laboratory practices as a criterion for selecting data: the case of bisphenol A. Environ Health Perspect. 117(3):309-15. doi: 10.1289/ehp.0800173. Epub 2008 Oct 22.

OECD (2013). Guidance document on developing and assessing adverse outcome pathways. Series on Testing and Assessment No. 184. Available at http://www.oecd.org/officialdocuments/publicdisplaydocumentpdf/?cote=env/jm/ mono(2013)6\&doclanguage $=$ en Appendix I. Collection of working definitions. Available at http://www.oecd.org/chemicalsafety/testing/49963576.pdf (accessed on 20 July 2017). 1

Regulation (EU) No 528/2012 of the European Parliament and of the Council of 22 May 2012 concerning the making available on the market and use of biocidal products.

Regulation (EC) No 1107/2009 of the European Parliament and of the Council concerning the placing of plant protection products on the market and repealing Council Directives 79/117/EEC and 91/414/EEC. OJ L 309.

Regulation (EC) No 1272/2008 of the European Parliament and of the Council of 16 December 2008 on classification, labelling and packaging of substances and mixtures

Schug TT, Johnson AF, Birnbaum LS, Colborn T, Guillette LJ Jr, Crews DP, Collins T, Soto AM, Vom Saal FS, McLachlan JA, Sonnenschein C, Heindel JJ. Minireview: Endocrine Disruptors: Past Lessons and Future Directions. Mol Endocrinol. 2016 Aug;30(8):833-47. doi: 10.1210/me.2016-1096. Epub 2016 Jul 19.

Scientific Committee on Emerging and Newly Identified Health Risks (SCENIHR). Memorandum on the use of the scientific literature for human health risk assessment purposes - weighing of evidence and expression of uncertainty. 19 March, 2012.

Segal D, Makris SL, Kraft AD, Bale AS, Fox J, Gilbert M, Bergfelt DR, Raffaele KC, Blain RB, Fedak KM, Selgrade MK, Crofton KM. (2015). Evaluation of the ToxRTool's ability to rate the reliability of toxicological data for human health hazard assessments. Regul Toxicol Pharmacol. 72(1):94-101.

Vandenberg LN, Hauser R, Marcus M, Olea N, Welshons WV. (2007) Human exposure to bisphenol A (BPA). Reprod Toxicol. 24(2):139-77. doi:10.1016/j.reprotox.2007.07.010

Vandenberg LN, Maffini MV, Sonnenschein C, Rubin BS, Soto AM. (2009) Bisphenol-A and the great divide: a review of controversies in the field of endocrine disruption. Endocr Rev. 30(1):75-95. doi: 10.1210/er.2008-0021. Epub 2008 Dec 12. Review.

Volkel W, and Colnot T CGFJDW. (2002). Metabolism and kinetics of bisphenol a in humans at low doses following oral administration. ed Chemical Research in Toxicology, 1281-1287.

Volkel W, Bittner N, and Dekant W. (2005). Quantitation of bisphenol A and bisphenol A glucuronide in biological samples by high performance liquid chromatographytandem mass spectrometry. 33: 1748-1757.

WHO/International Programme on Chemical Safety. (2002) Global assessment of the state of the science of endocrine disruptors (Damstra T, Barlow S, Bergman A, Kavlock R, Van Der Kraak G, eds.). 
http://www.who.int/ipcs/publications/new issues/endocrine disruptors/en/ (accessed on 20 July 2017). 